\title{
À quelle langue accède l'apprenant? Examen critique du traitement de l'oral dans les premières leçons de manuels de français langue étrangère pour débutants
}

\author{
Christian Surcouf et Anick Giroud (Lausanne)
}

\begin{abstract}
If one considers the recordings from textbooks of French as a foreign language, some typical spoken French phenomena appear not to reflect results provided by spoken French corpora. In order to have a better insight into this issue, we analyzed the recordings of the first three lessons of ten beginners' textbooks (1982-2012). In this article, we examine /a/ deletion (/зәvøləvwas/ vs. /3vølvwas/ 'je veux le voir'), reduction from /ty/ to /t/ before vowel (/tyabitu/ vs /tabitu/, 'tu habites où?'), and /l/ deletion in il(s)/elle(s) before consonant (/imãz/ vs. /ilmãz/, 'il mange'). We then compare our corpus's data with spoken French corpora. Finally, we discuss the possible origins of the discrepancies between both corpora.
\end{abstract}

\section{$1 \quad$ Introduction}

Il y a un quart de siècle, Séguin (1989: 124) s'interrogeait : «la principale tâche de la linguistique appliquée (à l'enseignement des langues) n'est-elle pas de mettre à jour nos connaissances de la langue dans son état actuel et de fournir ainsi aux concepteurs de matériel pédagogique un contenu descriptif qui soit didactiquement meilleur ? ». Vingt ans plus tard, Chambers (2009: 16) formule un souhait similaire en mentionnant le cas emblématique de la négation française et en imaginant à son tour qu' « on pourrait [...] envisager que la collaboration entre linguistes de corpus, auteurs de manuels, enseignants, et formateurs d'enseignants constitue une possibilité d'enrichir l'environnement de l'apprentissage du FLE ». C'est en somme à une telle entreprise que se consacre cet article en se focalisant sur trois phénomènes de l'oral dans les enregistrements des trois premières leçons de dix manuels de français langue étrangère (FLE) de niveau débutant. De quelle manière sont réalisés ces phénomènes oraux dans les enregistrements ? Reflètent-ils la réalité décrite par les analyses linguistiques des corpus du français parlé ? Afin de répondre à ces questions, on établira des comparaisons quantitatives entre les données de notre corpus d'enregistrements et celles issues des analyses linguistiques de corpus oraux. Nous montrerons que, malgré l'usage du terme " authentique » dans les avant-propos et les guides pédagogiques de tous les manuels de notre corpus, il existe de fortes disparités entre l'oral de ces enregistrements et la réalité des pratiques révélée par les corpus de français parlé. 


\section{$2 \quad$ Méthodologie}

\subsection{Les phénomènes retenus}

Nous avons retenu les trois phénomènes suivants :

1. La chute du /ə/. Dans l'énoncé /зəmapelsofidylak/ 'je m'appelle Sophie Dulac' le /ə/ de /3ə/ est maintenu, mais il ne l'est pas dans /3mapelsonja/ 'je m'appelle Sonia' (Accord 1, Berger/Spicacci 2000: 31/145, 6/142 $)^{1}$

2. La réduction du /ty/ à /t/ devant voyelle: /tagaje/ 't'as gagné' (Archipel 1, Courtillon/Raillard 1982a: 29/33)

3. La chute du /// dans /il/ et /عl/ devant consonne : /isõfьãse/ 'ils sont français ?' (Tempo 1, Bérard et al. 1996: 21)

\subsection{Le corpus utilisé}

Afin de fournir un aperçu documenté des phénomènes étudiés, nous avons retenu dix manuels de FLE couvrant une période de trente années (1982-2012), représentative de l'évolution méthodologique: avant les années 2000, avec la prédominance de l'approche dite communicative (5 manuels), puis avec l'apparition de l'approche actionnelle sous l'influence du Cadre européen commun de référence (CECR) à partir de 2001 (5 manuels également).

La durée totale des enregistrements analysés est d'environ 6h. L'analyse porte sur les enregistrements des trois premières « leçons ».

\begin{tabular}{|l|l|l|l|l|}
\hline \multicolumn{1}{|c|}{ Titre } & Année & \multicolumn{1}{c|}{ Auteurs } & \multicolumn{1}{c|}{ Leçons retenues } & \multicolumn{1}{c|}{ Durée } \\
\hline Archipel 1 & 1982 & Courtillon/Raillard & unités 1-3 & $48^{\prime}$ \\
\hline Cadences 1 & 1994 & Berger/Mérieux & séquences 0-3 & $24^{\prime}$ \\
\hline Tempo 1 & 1996 & Bérard et al. & unités 1-3 & $59^{\prime}$ \\
\hline Accord 1 & 2000 & Berger/Spicacci & unités 1-3 & $55^{\prime}$ (avec CD) \\
\hline Studio 601 & 2001 & Lavenne/Bérard & séquences 1-3 & $28^{\prime}$ \\
\hline Connexions 1 & 2004 & Mérieux/Loiseau & unités 1-3 & $44^{\prime}$ \\
\hline Rond-Point 1 & 2004 & Labascoule et al. & unités 1-3 & $25^{\prime}$ \\
\hline Ici 1 & 2008 & Abry et al. & unités 1-3 & $26^{\prime}$ \\
\hline Alter Ego+ 1 & 2012 & Berthet et al. & dossiers 0 et 1 leçons 1-3 & $46^{\prime}$ \\
\hline Zénith 1 & 2012 & Mimran/Poisson-Quinton & leçons 1-3 & $15^{\prime}$ \\
\hline
\end{tabular}

Tableau 1 : Les dix manuels constituant le corpus

Les enregistrements des leçons recensées dans le tableau ont été écoutés dans leur intégralité. Leurs thématiques s'articulent autour des salutations, de la présentation de soi, de la demande d'informations sur quelqu'un (description, gouts), ou plus généralement de renseignements sur la vie quotidienne (achats, sorties, démarches administratives, etc.). Y apparaissent non seulement des dialogues, mais aussi des exercices de phonétique ou de répétition, donnant

\footnotetext{
${ }^{1}$ La première mention de page de la référence renvoie à celle où l'enregistrement est utilisé, et, le cas échéant, la seconde - après / - celle où est fournie la transcription orthographique. Étant donné l'objectif poursuivi dans cet article, le contenu des enregistrements, transcrit en phonétique entre barres obliques, est donné en premier.
} 
parfois lieu à la lecture de mots isolés. Tous ces types d'exercice - à l'exclusion de la lecture des lettres de l'alphabet - ont été pris en compte. La durée des enregistrements variant d'un manuel à l'autre, notre analyse s'efforcera de montrer les tendances globales, même si elle donne un aperçu des différences - parfois considérables - entre manuels.

\subsection{La présentation adoptée}

La didactique du FLE s'inspirant des ouvrages de référence du français langue maternelle, on proposera dans le cas de la réduction des pronoms personnels un aperçu des descriptions fournies par de tels ouvrages. Nous ferons ensuite appel à certaines études linguistiques sur le phénomène traité et terminerons par une comparaison quantitative entre les résultats des corpus de français parlé et ceux des enregistrements de notre corpus.

\section{Présentation et analyse des phénomènes retenus}

\subsection{La chute du $/ /^{2}$}

Comme le rappelle Lyche dans le cadre de recherches au sein du projet de «Phonologie du français contemporain : usages, variétés et structure » (PFC), « la question du schwa [...] constitue sans nul doute l'une des épines de la phonologie du français » (Detey et al. 2010: 154). Étant donné la dimension avant tout didactique de notre propos, nous nous contenterons de donner ici un bref aperçu des difficultés de l'analyse linguistique du schwa, avant d'en venir aux principes simples que nous avons adoptés pour en recenser les occurrences dans les enregistrements de notre corpus.

Parler de «chute du /ə/ » laisse entendre que certaines variantes du français comprendraient des /a/ susceptibles de disparaitre sous certaines conditions. Toute la question réside en somme dans l'étalon à adopter pour identifier le schwa, que Léon présente ainsi :

Le E caduc doit son nom au fait qu'il peut tomber. On dira aussi bien Je sais que J'sais. [...] En français moderne, à l'oral, E muet de fin de mot (pote) sert à marquer la prononciation de la consonne finale, alors que celle-ci ne se prononce généralement pas si elle est en finale (pot).

(Léon 2007: 141)

On voit poindre ici le problème que pose l'identification du /ə/. À moins de considérer que /potə/ est la prononciation normale, déclarer que le «E muet» - le /ə/ - servirait à «marquer la prononciation de la consonne finale » - le /t/ -, c'est à priori prendre comme base de raisonnement ${ }^{3}$ des considérations relevant de la lecture et donc de l'écriture, où le graphème ' $\mathrm{e}$ ' - censé transcrire / $/$ / - indiquerait au lecteur que le ' $\mathrm{t}$ ' doit, contrairement au cas de pot, être $1 u^{4}$. Cet enchevêtrement des niveaux graphique et oral est en soi révélateur de la

\footnotetext{
2 En raison de la complexité du fonctionnement du schwa, nous nous limiterons ici aux explications de la littérature spécialisée (pour des explications issues d'ouvrages de référence, voir par exemple Arrivé et al. 1986: 518-519; Chevalier et al. 1964: 16-17; Riegel et al. 1994: 49; Riegel et al. 2009: 87-88).

3 Ailleurs, Léon (2007: 25) transcrit lui-même pâte [pa:t], sans le moindre [ə].

${ }^{4}$ Un enfant francophone encore non-alphabétisé ne différencie pas pot [po] de pote [pot] sur la base d'une supposée absence ou présence du graphème 'e', ou d'un « $E$ muet ». Diachroniquement, Morin (1978: 94) relève que : «word-final s[c]hwas were always reduced, and eventually disappeared completely in the 17 th century ». Une telle prononciation subsiste en partie en français méridional (cf. Blanche-Benveniste 2010: 22).
} 
prégnance de l'orthographe dans l'étude du schwa, phénomène pourtant oral $^{5}$. Morin nous met cependant en garde :

Most analyses of mute "e", [...], fail to make the distinction clearly, and are limited only to synchronic considerations, while depending upon the spelling (which indicates some of the history of the language) to define what is a mute "e".

(Morin 1978: 79)

Il paraitrait dès lors préférable d'opter pour une approche phonologique à l'instar de celle prônée par Côté et Morrison (2007: 161) «based on vowel alternation » (cf. également Detey et al. 2010: 154-155). Les auteurs mentionnent ainsi les alternances à l'intérieur d'un mot (cerise : [s(ә)ьіz]), entre deux frontières de mots (valse rapide: [vals(ә)ьарid]) ou d'affixes flexionnels ou dérivationnels (garderai [gакd(ә)ье], repartir [в(ә)раьтік]), en présence d'un clitique (ce bateau : [s(ə)bato]), et dans les noms composés : (garde-malade [gard(ə)malad]) (les exemples sont de Côté/Morrison 2007: 162). Cependant, des divergences existent entre linguistes sur la manière d'envisager les mêmes phénomènes. Pour Fougeron et al. (2001: 639) «a word containing schwa in its canonical form can be produced without the schwa ", explication que les auteurs illustrent à l'aide de «samedi » prononcé [samədi] ou [samdi]. Côté et Morrison (2007: 161) considèrent quant à eux que ce même mot se prononce systématiquement [samdi] dans l'oral spontané, avis partagé par Armstrong et Unsworth (1999: 135). Côté et Morrison (2007: 161) excluent également du domaine du schwa l'alternance $[\varepsilon] /[ə]$ : « in modern French: [E] alternates with zero [...] appelle [...] [apel] vs. appeler [...] [aple] », verbe dont la conjugaison apparait souvent dans les premières leçons des manuels pour débutants, précisément sous la forme /apəlõ/, /apəle/ (par exemple Accord 1, Berger/Spicacci 2000: 6, 10).

Comme l'illustre ce bref aperçu, définir le /ə/ reste «a thorny question » (Côté/Morrison 2007: 161), et le «standard » adopté repose souvent sur une représentation orale basée sur une lecture de l'orthographe. Dans une analyse minutieuse du phénomène, Bürki et al. (2011) tiennent compte de huit variables selon 18 dimensions, et concluent à l'importance déterminante de cinq variables seulement : 1) la vitesse de débit, 2) la position du schwa dans le mot, 3) la position du mot dans l'énoncé, 4) le nombre de consonnes dans la séquence consonantique, et 5) le respect du principe de sonorité ${ }^{6}$ dans cette séquence (Bürki et al. 2011: $3987)^{7}$.

En raison de la finalité beaucoup plus modeste de notre étude, le comptage des schwas de notre corpus a été effectué sur la base de notre propre intuition de locuteurs natifs du français

\footnotetext{
${ }^{5}$ Voir notamment le point de départ graphique proposé par Martinet (1974: 210). Il est clair que, comme le rappellent Durand et Eychenne (2004), pour la constitution d'un large corpus, le recours à la graphie est une étape indispensable. Toutefois l'outil de description ne doit pas être assimilé à l'objet décrit.

${ }^{6}$ Correspondant à l'échelle : « occlusive sourde $<$ occlusive sonore $<$ fricative sourde $<$ fricative sonore $<$ nasale $<$ liquide» (Bürki et al. 2011: 3983).

${ }^{7}$ Le corpus des auteurs étant « $24 \mathrm{~h}$ of radio-broadcasted news produced by 574 speakers » (Bürki et al. 2011: 3982), une telle conclusion doit être prise avec précaution quant à sa transférabilité au français spontané. Ce que reconnaissent les auteurs: "Whereas journalistic speech is certainly more natural than speech elicited in the laboratory, it differs from conversational speech in many respects. It is more formal and contains parts of prepared speech, which are probably more similar to read speech than to conversational speech » (Bürki et al. 2011: 3988).
} 
dit «standard $»^{8}$. Rappelons que les décomptes se basent sur l'hypothèse de l'existence d'un tel phénomène à des endroits précis de la chaine sonore, où une alternance entre présence et absence de /ə/ s'avère donc possible. En aucun cas, l'orthographe ne nous a servi de base. En définitive, la plupart de nos annotations se sont effectuées sans difficulté majeure ${ }^{9}$. Donnons trois exemples illustrant les principes suivis.

\begin{tabular}{|l|l|l|}
\hline \multicolumn{1}{|c|}{ Exemple } & \multicolumn{1}{|c|}{ Manuel } & \multicolumn{1}{c|}{ Décompte des /ə/ } \\
\hline $\begin{array}{l}\text { /predlagas/ } \\
\text { 'près de la gare' }\end{array}$ & $\begin{array}{l}\text { Ici } 1 \\
\text { (Abry et al. 2008: 25/116) }\end{array}$ & une chute (/dəla/ est possible) \\
\hline $\begin{array}{l}\text { /akotedəlaplas/ } \\
\text { 'à côté de la place' }\end{array}$ & idem & un maintien (/dla/ est possible) \\
\hline $\begin{array}{l}\text { /3əməsuireveje/ } \\
\text { 'je me suis réveillé' }\end{array}$ & $\begin{array}{l}\text { Tempol } \\
\text { (Bérard et al. 1996: 44/210) }\end{array}$ & $\begin{array}{l}\text { un maintien (/3məsui/ ou } \\
/ 3 ə m s u i / \text { sont possibles, pas } \\
/ *_{3} \text { msui/) }\end{array}$ \\
\hline
\end{tabular}

Tableau 2 : Illustration des principes suivis pour le décompte des schwas

\begin{tabular}{|c|c|c|c|c|c|}
\hline Maintien du/ə/ & & oui & & non & \\
\hline & Total & $\mathrm{N}$ & $\%$ & $\mathrm{~N}$ & $\%$ \\
\hline Archipel (1982) & 183 & 105 & 57 & 78 & 43 \\
\hline Cadences (1994) & 37 & 31 & 84 & 6 & 16 \\
\hline Тетро (1996) & 215 & 165 & 77 & 50 & 23 \\
\hline Accord (2000) & 163 & 143 & 88 & 20 & 12 \\
\hline Studio (2001) & 85 & 66 & 78 & 19 & 22 \\
\hline Connexions (2004) & 206 & 177 & 86 & 29 & 14 \\
\hline Rond-Point (2004) & 170 & 118 & 69 & 52 & 31 \\
\hline Ici (2008) & 127 & 94 & 74 & 33 & 26 \\
\hline Alter Ego $+(2012)$ & 270 & 240 & 89 & 30 & 11 \\
\hline Zénith (2012) & 74 & 69 & 93 & 5 & 7 \\
\hline Total général & 1530 & 1208 & $79 \%$ & 322 & $21 \%$ \\
\hline
\end{tabular}

Tableau 3 : La répartition des /ə/ dans les trois premières leçons des dix manuels

Comme le montrent les analyses, le maintien des /a/ s'avère largement majoritaire dans l'ensemble des manuels (79\%). Comparons maintenant avec les données recueillies en 1989

\footnotetext{
${ }^{8}$ Voir les réflexions de Lyche sur la prononciation du « français de référence » dans Detey et al. (2010: 143).

${ }^{9} \mathrm{Il}$ est clair que parmi les phénomènes retenus ici, c'est le schwa qui s'avérait le plus délicat à traiter. Le corpus ayant été divisé en deux, chaque auteur a annoté sa partie et a fait appel à l'autre en cas d'incertitude.
} 
par Hansen (2000) à l'issue de conversations spontanées et d'entretiens avec des «Parisiens cultivés » (huit adultes entre 40-55 ans, et seize jeunes entre 15-25 ans).

\section{Notre corpus $(\mathrm{N}=1530)$}

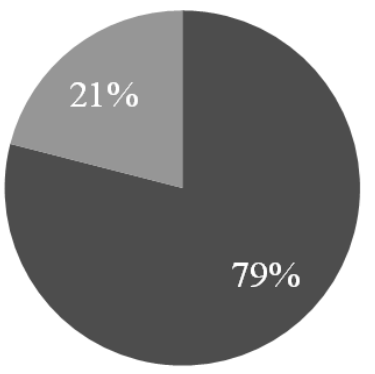

\section{Corpus de Hansen $(\mathrm{N}=4969)$}

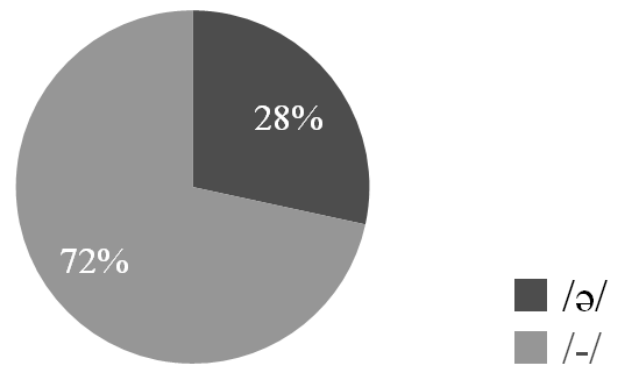

Figure 1 : Comparaison du maintien du /a/ avec la synthèse calculée à partir de Hansen (2000: tableau 1) ${ }^{10}$

Manifestement - et sans surprise pour le locuteur natif les ayant écoutés -, les énoncés des enregistrements des trois premières leçons des manuels de notre corpus ne reflètent pas la réalité décrite par Hansen ${ }^{11}$. Comme l'illustre la figure 2, paradoxalement, c'est le manuel le plus ancien - Archipel 1 (1982) - qui, avec 57\% de maintien (soit, malgré tout, le double du corpus de Hansen), s'avère le plus proche du français oral. Les deux manuels les plus récents (Alter Ego+ 1 et Zénith 1) sont les plus éloignés de cette réalité (89\% et 93\%).

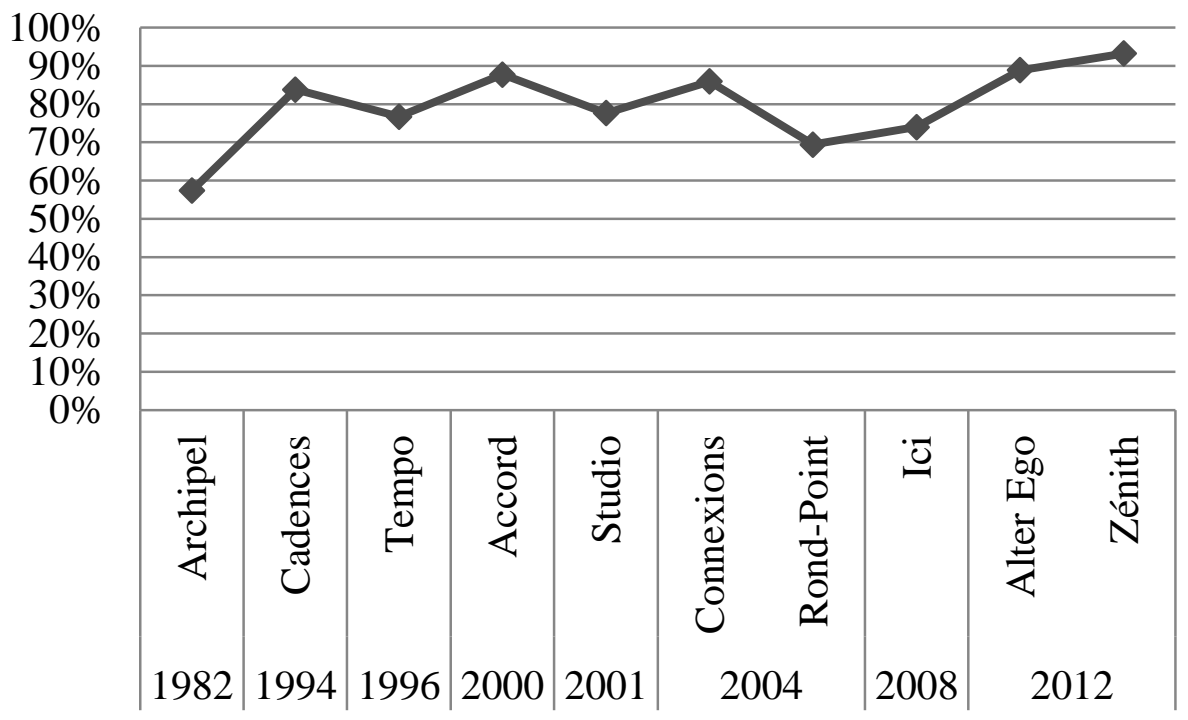

Figure 2 : Pourcentages de maintien du /ə/ dans les dix manuels

En définitive, comme le remarque Lyche (Detey et al. 2010: 160) « les ouvrages de FLE [...] jettent sur la phonologie un regard relativement conservateur $»$.

\subsection{La réduction $\mathrm{de} / \mathrm{ty} / \mathrm{à} / \mathrm{t} /$}

10 Les données «monosyllabe, «syllabe initiale» et «syllabe médiane», ainsi que des «conversations spontanées » et des « entretiens » ont été fusionnées.

11 L'étude de Fougeron et al. (2001: 640) sur 10 locuteurs suisses (20-30 ans) établit à 36\% le maintien de /a/ $(\mathrm{N}=543)$. 
La linguistique de l'oral semble s'être peu attardée sur le phénomène de l'élision du $t u$. Dans un article consacré aux «sujets de deuxième personne à l'oral» (au sein de CorpAix), Cappeau (2004: 76-77) remarque qu'il «est parfois réalisé par la consonne seulement ([...] t'as $v u$ ) mais les transcriptions que nous utilisons restituent la forme complète (tu as $v u$ ) ». François (1974: 642), dans son corpus recueilli en 1964 en région parisienne, relève quant à elle : " Le pronom sujet $t u$ (132 occurrences) se réalise [ty] ou [t]. Devant consonne, on trouve généralement [ty] et devant voyelle, généralement $[\mathrm{t}]$ ». Quelles fréquences d'usage se dissimuleraient derrière les adverbes parfois (Cappeau) et généralement (François)? Les ouvrages de référence évoquent eux aussi les variantes /ty/-/t/, la seconde étant toujours supposée renvoyer à une dimension « familière » :

1) Dans l'usage familier, $t u$ prend la forme $t^{\prime}$ ([t]) dans les mêmes conditions [que celle de je] : t'enrages, t’hésites (mais tu harcèles) (Arrivé et al. 1986: 499)

2) Dans la langue parlée familière, tu se réduit souvent à [t] devant voyelle (Grevisse/Goosse 2008: 842)

3) L'usage familier prononce [...] $t u$ [t] devant voyelle (t'arrives ?) (Riegel et al. 1994: 200; 2009: 368)

Ce que confirment les dictionnaires :

4) Dans la langue familière ou parlée, $t u$ s'élide en $t$ ' devant une voyelle ou un $h$ muet ( $t$ 'as raison, t'en fais pas, t'es folle, t'hésites ?) (Le dictionnaire du français) (Rey-Debove 1999: 1048) (cf. également Rey-DeboveRey 2009)

Cette dimension familière est également évoquée dans deux ouvrages linguistiques importants dans la problématique du français parlé :

5) le $[y]$ de $t u,[\ldots]$ disparait, en usage familier, quand le verbe suivant commence par une voyelle (Gadet 1989: 104)

6) Le [y] du pronom $t u$ s'élide dans les prononciations familières, comme on le voit chez un Parisien de vingt-six ans dans une conversation entre amis : tu auras [tora] - tu es [te] - tu as [ta] - tu allumes [talym] (Blanche-Benveniste 2010: 31)

Cette unanimité à reconnaitre dans l'élision $\mathrm{du} / \mathrm{y} /$ une dimension « familière » n'est pas exempte de problèmes. Y aurait-il en français contemporain (mais voir déjà Damourette/Pichon 1911-1940: 274 §2332) des «conversations entre amis » de « vingt-six ans » se tutoyant (6), qui ne seraient pas par nature «familières » si «le français familier, [est] celui dont chacun est porteur dans son fonctionnement quotidien, dans le minimum de surveillance sociale » (Gadet 1989: 3) ${ }^{12}$ ou encore « ce qui [...] s'emploie dans des situations

\footnotetext{
12 Il s'agit pour Gadet de définir ici «le français ordinaire », qu'elle considère comme proche du «français familier $»$.
} 
de spontanéité amicale » (Rey-Debove 1999: XI) ${ }^{13}$ ? La variante /t/ ne serait-elle pas dès lors celle la plus appropriée à ce genre de situation ${ }^{14}$ ?

L'analyse des corpus s'avère donc fondamentale pour permettre d'établir ce que pourrait signifier le qualificatif «familier » utilisé dans toutes ces citations ${ }^{15}$. Étant donné que nous n'avons trouvé aucune donnée quantitative sur ce phénomène, nous avons mené une analyse des transcriptions de sept corpus du laboratoire Clapi du français oral spontané en situation de dialogue à deux ou quatre personnes dans des conversations entre amis, en famille, ou lors d'une réunion de travail entre collègues (Bruxelles et al. 2002; Gatti 2008; Otsuka 2006; Thévenon/Tommasini 2008; Traverso 1985-1986) ${ }^{16}$. Sur les 3h52' d'enregistrements, on obtient 209 occurrences de $t u+$ voyelle. La répartition est la suivante :

\begin{tabular}{|l|l|l|}
\hline Variante & \multicolumn{1}{|c|}{$\mathrm{N}$} & \multicolumn{1}{c|}{$\%$} \\
\hline$/$ ty/ & 26 & $12 \%$ \\
\hline$/ \mathrm{t} /$ & 183 & $88 \%$ \\
\hline total & 209 & $100 \%$ \\
\hline
\end{tabular}

Tableau 4 : Résultats de l'analyse de corpus Clapi

Les corpus s'étendent de 1985 à 2008 et émanent de locuteurs de la classe moyenne, âgés de 22 ans à 65 ans. Il ne s'agit plus là d'une tendance à l'utilisation de /t/ devant voyelle, mais bel et bien de la configuration par défaut, où l'articulation /ty/ s'avère largement minoritaire $(12 \%)$, puisque sept fois moins fréquente. En d'autres termes, on pourrait s'interroger sur la fréquence si basse de ce genre d'usage attesté du français parlé dans les enregistrements des manuels de FLE, où les proportions sont inversées, comme le montre la figure 3.

\section{Notre corpus $(\mathrm{N}=127) \quad$ Analyses de corpus Clapi $(\mathrm{N}=209)$}
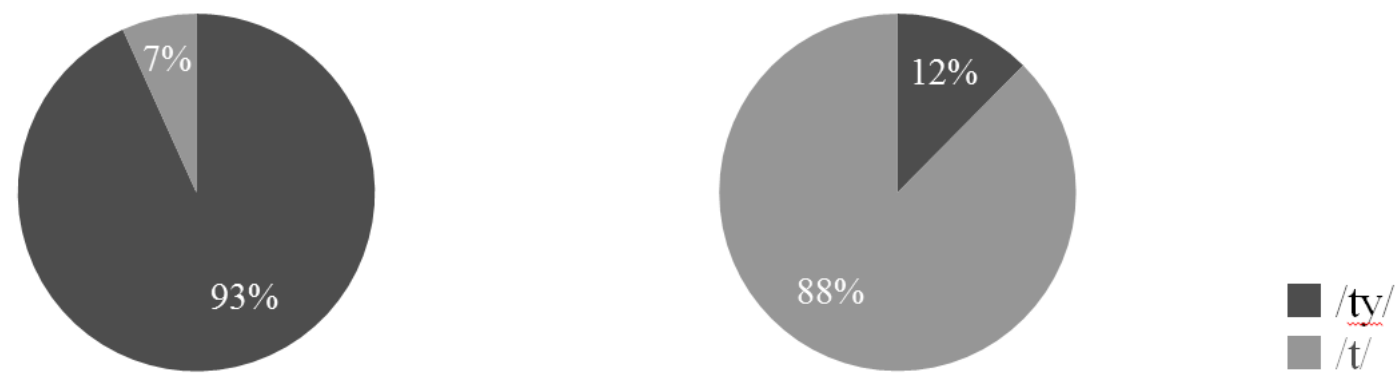

Figure 3 : Comparaison entre les données de notre corpus pour tu et celles de Clapi

13 Comme le remarque Rouayrenc (2010: 284) le « fait de distinguer trois registres [courant, soutenu, familier] est certes commode, mais la distinction, d'une part, n'est fondée ni sur des critères précis, ni sur des enquêtes systématiques de la réalité souvent plus complexe de l'oral où les divers registres interfèrent » (cf. également Gadet 1989: 18-20).

14 Si l'on peut admettre que le $t u$ s'utilise dans certains milieux entre deux inconnus dès la première rencontre, il s'agit là d'usages marginaux « entre élèves, dans certains corps professionnels, ou communautés religieuses, sportives, etc. » (Charaudeau 1992: 123-124).

15 En ce sens nous rejoignons la réflexion de Lodge :

language varieties, particularly spoken ones, are inherently variable - as a rule and not as an exception - and [...] there are no pure varieties of contemporary French, merely quantitative differences in the distribution of key language variables. This means that labels like français populaire, français familier, français régional can be profoundly misleading, for they imply the existence of discrete sociolinguistic varieties rather than an observer's arbitrary division of variable linguistic continua. (Lodge 1993: 232)

16 Les vidéos sont disponibles en ligne au lien indiqué en bibliographie. 


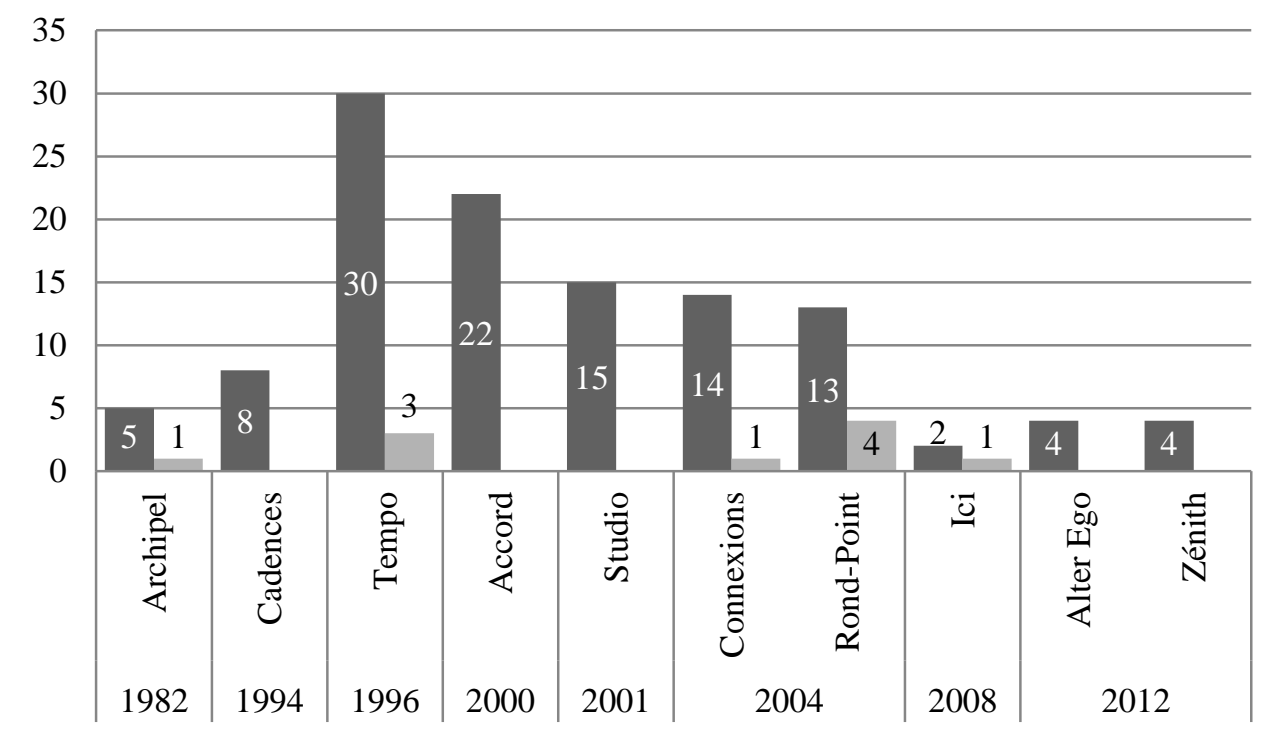

Figure 4 : La répartition des variantes /ty/-/t/ dans notre corpus

La moitié des manuels n'utilisent jamais la variante /t/, et s'avèrent dès lors en porte-à-faux avec l'usage du français oral. Remarquons que les auteurs de Tempo 1 sensibilisent explicitement à l'existence de la variante $/ \mathrm{t}+\mathrm{V} /$ dans un exercice de compréhension orale. L'apprenant est invité à réécouter le dialogue et cocher la case de ce qu'il a entendu en choisissant parmi les deux variantes, rapprochées pour l'occasion :

\begin{tabular}{|c|c|c|}
\hline \multicolumn{2}{|c|}{ Questions de « compréhension orale » sur le dialogue } & Transcription en fin de volur \\
\hline \multicolumn{2}{|c|}{$\begin{array}{l}\text { 5. Écoutez à nouveau le premier dialogue. } \\
\text { a. Qu'est-ce que vous avez entendu? }\end{array}$} & \multirow{5}{*}{$\begin{array}{l}\text { - Tu t'appelles comment? } \\
\text { - Catherine. } \\
\text { - Moi, c'est Olivier. T'es en droit? } \\
\text { - Non, j'fais médecine. } \\
\text { - Moi aussi. T'es en première année } \\
\text { - Ouais... } \\
\text { - T'as trouvé une chambre? } \\
\text { - Ben non... }\end{array}$} \\
\hline $\begin{array}{l}\square \mathrm{Tu} \\
\square \text { Vous }\end{array}$ & $\begin{array}{l}\square \text { Oui } \\
\square \text { Ouais }\end{array}$ & \\
\hline $\begin{array}{l}\square \text { Tu t'appelles comment? } \\
\square \text { Comment est-ce }\end{array}$ & $\begin{array}{l}\square \text { Tu as trouvé une chambre? } \\
\square \text { T'as trouvé une chambre? }\end{array}$ & \\
\hline que tu t'appelles? & Au revoir & \\
\hline $\begin{array}{l}\text { T'es en première année? } \\
\text { Tu es en première année? }\end{array}$ & $\begin{array}{l}\square \text { Salut } \\
\square \text { Tchao }\end{array}$ & \\
\hline
\end{tabular}

Figure 5 : Exemple de sensibilisation à la variante /t/ dans Tempo 1 (Bérard et al. 1996: 42/209)

\subsection{La réduction $\mathrm{de} / \mathrm{il} / \mathrm{et} / \varepsilon \mathrm{l} / \mathrm{à} / \mathbf{i} / \mathrm{et} / \boldsymbol{\varepsilon} /$}

S'il semble logique de considérer que, par son sémantisme, tu représente le pronom par excellence de la situation dite "familière », tel n'est pas le cas pour il(s) et elle(s). Contrairement à la réduction de $t u$, la linguistique de corpus s'est davantage penchée sur ce phénomène. Commençons notre tour d'horizon des ouvrages de référence :

7) $I l$ et $i l s$ sont couramment prononcés [i] devant consonne : il (s) démarre(nt), [idemar]. La réduction de elle(s) comme forme conjointe à $[\varepsilon]$ est familière : elle(s) démarre $(n t)$ [ $\varepsilon$ demar $]$ (Arrivé et al. 1986: 499)

8) L'usage familier prononce [...] il [i] devant initiale consonantique (I (l) vient), ils [iz] devant initiale vocalique $(I(l)$ s arrivent $)[\ldots]$ et même de façon très relâchée, réduit $[. .$.$] elle$ sujet à [ع] ou [a] (Riegel et al. 1994: 200) 
9) Devant une consonne, il et ils se prononcent [il] dans la langue soutenue, [i] dans la langue familière. Devant voyelle : au singulier, [il] ; au pluriel, [ilz] dans la langue soutenue, [iz] dans la langue familière. [...] [a], prononciation pop[ulaire] dans diverses régions, pour elle, elles (Grevisse/Goosse 2008: 842)

Le nouveau Petit Robert (2010) précise : «Dans la prononciation familière : il dit [idi]; ils ont dit [izõdi] » mais n'offre aucune remarque sur la prononciation de elle(s). Le Dictionnaire du français - destiné à un public de FLE - transcrit ces deux pronoms [il] et [عl] et n'évoque aucune variante. Manifestement le traitement de $i l(s)$ et elle(s) diverge selon les auteurs, qui semblent trouver plus acceptable la réduction du premier («couramment», "langue familière ", " usage familier ») que du second ("familière », " populaire », " très relâchée »). Ce que confirment les observations de Gadet (cf. également Morin 1988: 263-264) :

La chute du 1 du pronom $i l$, fréquemment dénoncée comme changement (stigmatisé) en cours, remonte en fait à l'ancien français. Elle est, en français standard, réservée à la position préconsonantique [...] De façon semble-t-il plus récente, le comportement de 1 dans le pronom elle est parallèle : [keskedi]

(Gadet 1989: 103-104)

Dans la première moitié du siècle dernier, Damourette/Pichon (1911-1940: 279 §2336) relevaient déjà que «Dans la prononciation courante, il arrive fréquemment que elle se réduise à [è], tant dans la parlure optimale que dans celle du menu peuple ». Quant à $i l(s)$, les deux auteurs précisaient (cf. également Blanche-Benveniste 2010: 23) :

Il importe de tenter de décrire avec bonne foi l'usage actuel que les auteurs n'ont pas toujours bien réussi à préciser. De nos jours, dans le français normal, c'est-à-dire dans la bourgeoisie cultivée de Paris, en parlant on prononce toujours ou à peu près toujours [i] devant consonne

(Damourette/Pichon 1911-1940: $277 \S 2335)^{17}$

Qu'en est-il des données statistiques proposées par les corpus oraux ? Parviendraient-elles à nous affranchir de ces libellés («familier », «populaire », " normal », etc.) plus ou moins discutables (cf. note 13) ? Dans son étude publiée en 1975, sur 50 conversations (d'une longueur moyenne de 30 minutes) de locuteurs cultivés de la classe moyenne parisienne, Malécot (1975: 171-172) relève que trois $i l$ sur quatre se prononcent /i/ devant consonne et semi-consonne $(76,8 \% ; 71,8 \%)^{18}$. L'auteur pressentant qu'un tel résultat constituerait «a surprise to many » avertit ses lecteurs : "We understand that this may be difficult for some purists to accept. Indeed most of us are convinced that we speak quite differently than we actually do » (1975: 171-172). Ashby (1984) établit quant à lui son corpus à partir de 26 locuteurs tourangeaux de classes sociales différentes et répartis en deux groupes générationnels de 14-21 et 51-64. Ses résultats confirment amplement ceux de Malécot :

\begin{tabular}{|l|l|c|l|c|l|}
\multicolumn{1}{|c|}{$/ 1 /$ ? } & sans & $\%$ & avec & $\%$ & Total \\
\hline il $(s)$ & 2714 & $\mathbf{8 1 \%}$ & 621 & $19 \%$ & 3335 \\
\hline elle $(s)$ & 117 & $\mathbf{2 9 \%}$ & 291 & $71 \%$ & 408 \\
\hline
\end{tabular}

17 Ailleurs les auteurs considèrent que «L'emploi de [il] devant consonne est un orthographisme ; dans la lecture, beaucoup de gens de toutes classes l'emploient, à tort d'ailleurs. » (Damourette/Pichon 1911-1934: 524 $\S 1530)$; à ce propos voir Gadet (2007: 46-47).

18 Le nombre total d'occurrences n'est pas fourni. 
Tableau 5 : Il(s), elle(s) et leurs variantes /i/, / $/$ / (Ashby 1984) ${ }^{19}$

Dans une étude récente, mais d'amplitude plus modeste, Brognaux/Avanzi (2015: 4) relèvent seulement $5,76 \%$ de /il/ en français conversationnel sur un total de 120 locuteurs issus de trois zones géographiques différentes (Belgique, France, Suisse).

Dans notre corpus, sur les 80 occurrences de elle(s) devant (semi-/)consonne, aucune réduction n'a été relevée. Le $i l(s)$ est en revanche réduit à /i/ dans trois manuels, soit à 14 reprises sur les 250 occurrences devant (semi-/)consonne : deux dans Archipel 1 (1982), six dans Tempo 1 (1996) et six dans Rond-Point 1 (2004). En voici trois exemples :

10) /idezœnalakãtin/ 'il déjeune à la cantine' (Archipel 1, Courtillon/Raillard 1982b: 37)

11) /isõfьãse/ [...] /ipaslfıãse/ 'Ils sont français ?' 'Ils parlent français ?' (Tempo I, Bérard et al. 1996: 21/208)

12) /ivisœl/ 'il vit seul' (Rond-Point 1, Labascoule et al. 2004: 19/68)

\section{Notre corpus $(\mathrm{N}=228) \quad$ Corpus de Brognaux et Avanzi (2015) 20}
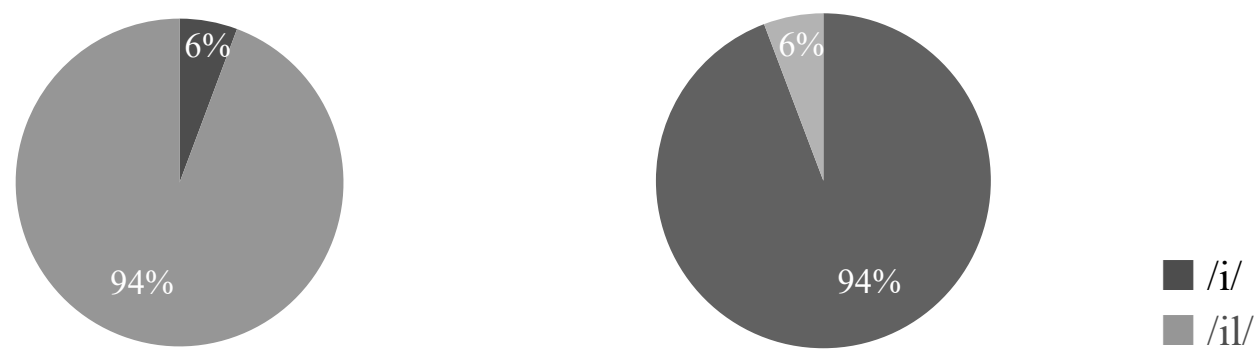

Figure 6 : Réduction de /il/ à /i/ dans les clitiques il(s)

Si les variantes /il/ et /i/ coexistent, /i/ domine très largement dans tous les corpus oraux mentionnés, quelle que soit la date de l'étude. Les enregistrements des manuels de notre corpus donnent toutefois une représentation inversée de la répartition des deux variantes utilisées en français parlé.

\section{Conclusion}

En dépit des souhaits de Séguin (1989: 124) et de Chambers (2009: 16) évoqués dans l'introduction, comme le montre notre analyse, même les manuels les plus récents (Alter Ego+1 et Zénith 1 2012) offrent dès leurs premières leçons une vision de la langue orale divergeant considérablement des données des corpus oraux. Ainsi, même sur une langue comme l'anglais dont la description linguistique s'avère extrêmement vaste, Gilmore (2007: 98) constate que «the language presented to students in textbooks is a poor representation of the real thing ». Comment expliquer cette divergence?

\footnotetext{
19 Les données de Ashby (1984: 7) fusionnent toutes les réductions de il(s), elle(s), que ce soit devant consonne, semi-consonne, pause ou voyelle. Ashby (1984: 8) indique dans le tableau 3 le nombre d'occurrences selon les environnements (consonne, etc.) sans toutefois distinguer il(s) et elle(s), si bien qu'il n'est pas possible de connaitre le pourcentage exact de $i l(s)$ devant (semi-/)consonne. Des calculs au prorata l'évalueraient jusqu'à 90\%. Des tendances analogues sont également mentionnées dans des études plus récentes (cf. Armstrong 2001: $70)$.

20 Le nombre total d'occurrences pour le français conversationnel n'est pas fourni. Les 494 occurrences mentionnées fusionnent sans en détailler la répartition le français lu $(96,15 \%$ de /il/), et le français conversationnel $(5,76 \%$ de $/ \mathrm{il} /)$.
} 
Une telle question renvoie à la problématique - ancienne - de l'intégration des documents authentiques dans les manuels de langue. Problématique d'autant plus difficile, qu'elle touche ici la dimension orale. Il semblerait que les résultats potentiellement utiles, issus des recherches en didactique ou de domaines connexes ne parviennent guère à se frayer un chemin jusqu'aux manuels ou aux pratiques de classe (cf. Gilmore 2007: 112), et restent la plupart du temps méconnus des concepteurs et des éditeurs. Comme le signale Gilmore (2007: 112), il est possible que les retournements importants («wild pendulum swings ») des cinquante dernières années dans l'enseignement des langues aient engendré chez les enseignants (ainsi que les concepteurs et les éditeurs) une certaine réticence vis-à-vis de toute nouvelle vogue didactique ( «yet another fashionable trend»), rendant dès lors délicate l'intégration des résultats de nouvelles recherches.

Cependant, on pourrait idéalement imaginer que les divergences mises au jour par notre étude entre les enregistrements des manuels et les corpus oraux résultent, de la part des concepteurs de manuels, d'une stratégie didactique délibérée, censée faciliter l'apprentissage. Un tel choix demanderait cependant à être argumenté et concrètement démontré ${ }^{21}$. Par exemple, le maintien $\mathrm{du} / \mathrm{\partial} /$, dont le taux est trois fois supérieur à celui des corpus oraux $(79 \%$ contre $28 \%$ ) permettrait-il une meilleure compréhension, une meilleure appropriation, une meilleure mémorisation ? Serait-il plus facile de comprendre (a) /zəsuifatige/ que (b) / /uifatige/ en dépit du fait que (a) ne sera guère entendu en dehors des enregistrements des manuels ? S'agit-il vraiment là d'une stratégie didactique délibérée ? Probablement pas. En témoigneraient certains indices, trouvés par exemple dans Cadences 1, où les intentions des concepteurs se voient trahies par la diction des comédiens dans les enregistrements.

À titre d'illustration, examinons l'exercice suivant, où l'apprenant doit biffer les « lettres muettes ».

\section{OO Lisez ces phrases et barrez les lettres muettes. Écoutez pour contrôler.}

Exemple : Ellæxs'appellø Gemma.

1 Elles sont françaises.

2 Vous êtes autrichienne.

3 Regardez le tableau.

4 Quelle est votre profession?

5 Ils ont l'air très content.

6 C'est une journaliste célèbre.
7 Quelle est l'adresse de Catherine?

8 C'est le père de Julie avec notre professeur.

9 Elle est trop petite.

10 Il habite à Paris, près de la gare du Nord.

\section{Les finales des mots}

Le e final ne se prononce pas. un(e) cart(e) ell( s'appell(

En général, les consonnes finales $(d, p, s, t, x, z)$ ne se prononcent pas. $\operatorname{tar}(\mathrm{d}$ - tro(p)- trè(s) - tô(t) - pri(X)- ri(z)

N.B. IIS répèt ent [repet] Elle(s) parlent [parl]

Figure 7 : Cadences 1 (Berger/Mérieux 1994a: 23)

\footnotetext{
21 Or, comme nous l'avons déjà mentionné dans l'introduction, l'examen des avant-propos et des guides pédagogiques révèle au contraire l'usage récurrent du terme «authentique » dans tous les manuels de notre corpus, sans que cette «authenticité » soit effective dans l'oral présenté, manifestement toujours fabriqué (Giroud et Surcouf 2016).
} 
L'écoute des enregistrements met en évidence des disparités flagrantes (en 3, 6, 8 et 10) entre l'intention des concepteurs ${ }^{22}$ apparaissant explicitement dans le corrigé du guide pédagogique à gauche dans la figure 8 , et la manière dont elle a effectivement été réalisée par les comédiens (à droite).

Le corrigé fourni dans le guide pédagogique

C 1 Elles sont françaises.

2 Vous êtes autrichienne.

3 Regardez le tableau.

4 Quelle est votre profession?

5 Ils ont l'air très contents.

6 C'est une artiste célèbre.

7 Quelle est l'adresse de Catherine?

8 C'est le père de Julie avec notre professeur.

9 Elle est trop petite.

10 Il habite à Paris, près de la gare du Nort.

\section{La prononciation réelle des comédiens}

3 /вәgardelatablo/

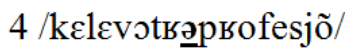

6 /sctynastistaselelebs/

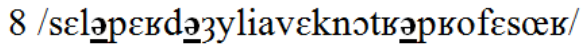

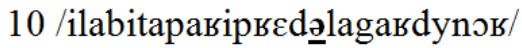

Figure 8 : Cadences 1 (Berger/Mérieux 1994a: 23; Berger/Mérieux 1994b: 15)

En 3, /вәgавdeltablo/, en 8 /sclpesd3yli/ et en 10 /рьєdlagaь/ sont les énoncés souhaités par les concepteurs mais non-réalisés par les comédiens, bien que la prononciation des segments en gras ne pose aucune difficulté, et correspondrait au français parlé ${ }^{23}$.

$\mathrm{Si}$, en l'absence d'information sur le processus d'élaboration des manuels, les indices discutés ici ne peuvent être extrapolés à tous les enregistrements, ils n'en révèlent pas moins qu'en dernier ressort, il se peut que la prestation orale des comédiens détermine la nature du résultat final. Ainsi la lecture des énoncés proposés par écrit aboutirait-elle au maintien de davantage de /a/ que durant toute production orale spontanée. Une telle hypothèse se verrait confirmée par les analyses de Hansen (2000: tableau 1) montrant un taux de maintien en lecture de $67 \%$ $(\mathrm{N}=3687)$ contre $28 \%(\mathrm{~N}=4969)$ dans l'oral spontané $(73 \%$ contre $36 \%$ dans l'étude de Fougeron et al. 2001: 640). De tels taux sont proches des 78\% (N=1717) de notre corpus. En d'autres termes, il semblerait que le français des enregistrements corresponde davantage aux canons de l' 'écrit oralisé' qu'à ceux de l'oral spontané.

Il est également possible qu'un autre facteur interfère, susceptible de conduire - plus ou moins consciemment - les comédiens à soigner leur diction dans ce qu'ils savent pertinemment constituer une «langue adressée aux étrangers ${ }^{24}$ ». Dans son étude sur l'enseignement des langues par des natifs (le tchèque, l'allemand et l'anglais), Henzl (1979: 164) observe ainsi :

A comparison of the samples brought evidence that speech directed to the foreign language learners was marked by phonemically more accurate standard pronunciation than speech to native

\footnotetext{
${ }^{22}$ Intention difficile à réaliser en certains points. La séquence /sts/ en 6 serait délicate à prononcer. En 4 et 8 , la

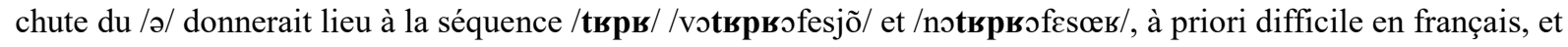
couramment prononcée /v\notpвəf.../, sans /в/ final à votre et notre (cf. Armstrong 2001: 67s).

${ }^{23}$ Aucun des comédiens n'a d'accent méridional qui justifierait - du moins aux oreilles d'un natif - le maintien des /a/ dans ces positions.

${ }^{24}$ Nous traduisons ici l'expression « foreigner talk », définie par Ferguson (1975: 2).
} 
speakers. [...] teachers spoke slowly to the foreigners and increased the rate of their speech when they talked to listeners of equal linguistic competence; [...]

The lower rate of the speech [...] was further associated with distinct patterns of word segmentation and accentuation. [...] the classroom talk was marked by a considerably higher frequency of unreduced vowels and consonantal clusters.

(Henzl 1979: 164)

Un tel comportement pourrait (inconsciemment) être adopté par les comédiens lors des enregistrements. Remarquons cependant, que si la langue adressée aux étrangers «entails reduced speaking rates with increased pausing, greater loudness, more marked stress, and clearer enunciation. [...] these adjustments are not always helpful to L2 listeners, and popular beliefs about their effectiveness are sometimes mistaken » (Munro 2013: 2733). En définitive, que les phénomènes mis en évidence par notre étude résultent d'une volonté des concepteurs et des éditeurs des manuels ou de la lecture (trop) soignée des comédiens, le décalage avec le français oral spontané ne constituerait pas nécessairement une aide pour l'apprenant. Même en supposant que, dans l'instant de l'exercice d'écoute, de tels artifices s'avéraient effectivement facilitateurs - ce qu'il faudrait prouver -, que deviendra l'apprenant face à la langue telle qu'elle se parle (et telle que la linguistique de corpus la décrit) ? Par leur nature même, les enregistrements ne devraient-ils pas sensibiliser les apprenants à l'oral tel qu'il se pratique plutôt qu'aux canons de la lecture?

De telles disparités entre les enregistrements et le français parlé - mises en évidence par notre étude - viendraient-elles à s'atténuer voire à disparaitre avec la naissance d'un nouveau paradigme, appelé de ses vœux par Gilmore (2007: 113), venu des avancées de l'apprentissage sur corpus (cf. Boulton/Tyne 2014)? Probablement, mais l'usage pédagogique de corpus oraux semble complexe à mettre en place. En attendant, l'apprenant de FLE des premières leçons continuera vraisemblablement à être soumis à la diction « endimanchée $»^{25}$ des comédiens...

\section{Bibliographie}

Armstrong, Nigel (2001): Social and stylistic variation in spoken French: a comparative approach. Amsterdam: Benjamins.

Armstrong, Nigel/Unsworth, Sharon (1999): "Sociolinguistic variation in southern French schwa”. Linguistics 37/1: 127-156. doi: 10.1515/ling.1999.001.

Arrivé, Michel/Gadet, Françoise/Galmiche, Michel (1986): La grammaire d'aujourd'hui: guide alphabétique de linguistique française. Paris: Flammarion.

Ashby, William J. (1984): "The Elision of /1/ in French Clitic Pronouns and Articles". In: Pulgram, Ernst (ed.): Romanitas: Studies in Romance Linguistics 4. Ann Arbor, University of Michigan Press: 1-16.

Blanche-Benveniste, Claire (1982): "La escritura del lenguaje dominguero". In: Jerreiro, Emilia/Gómez Palacio, Margarita (eds.): Nuevas perspectivas sobre los procesos de lectura y escritura. Mexico, Siglo XXI: 247-270.

Blanche-Benveniste, Claire/Martin, Philippe (2010): Le français. Usages de la langue parlée. Leuven/Paris: Peeters.

\footnotetext{
${ }^{25}$ Nous nous inspirons du terme de Blanche-Benveniste (1982).
} 
Boulton, Alex/Tyne, Henry (2014): Des documents authentiques aux corpus: démarches pour l'apprentissage des langues. Paris: Didier.

Brognaux, Sandrine/Avanzi, Mathieu (2015): "Sociophonetics of phonotactic phenomena in French". Proceedings of the International Conference on Phonetic Sciences: 1-5.

Bruxelles, Sylvie et al. (2002): Réunion de conception en architecture - mosaic. Lyon: Clapi. http://clapi.ish-lyon.cnrs.fr/ [07.10.2015].

Bürki, Audrey et al. (2011): "What affects the presence versus absence of schwa and its duration: A corpus analysis of French connected speech". Journal of the Acoustical Society of America 130/6: 3980-3991.

Cappeau, Paul (2004): «Les sujets de deuxième personne à l'oral ». Langage et société 108: 75-90. doi: 10.3917/1s.108.0075.

Chambers, Angela (2009): «Les corpus oraux en français langue étrangère: authenticité et pédagogie ». Mélanges Crapel 31: 15-33.

Charaudeau, Patrick (1992): Grammaire du sens et de l'expression. Paris: Hachette.

Chevalier, Jean-Claude/Blanche-Benveniste, Claire/Arrivé, Michel/Peytard, Jean (1964): Grammaire Larousse du français contemporain. Paris: Larousse.

Côté, Marie-Hélène/Morrison, Geoffrey Stewart (2007): "The nature of the schwa/zero alternation in French clitics: experimental and non-experimental evidence". Journal of French Language Studies 17/2: 159-186.

Damourette, Jacques/Pichon, Édouard (1911-1934): Des Mots à la Pensée, Tome IV. Paris: D'Artrey.

Damourette, Jacques/Pichon, Édouard (1911-1940): Des Mots à la Pensée, Tome VI. Paris: D'Artrey.

Detey, Sylvain/Durand, Jacques/Laks, Bernard/Lyche, Chantal (eds.) (2010): Les variétés du français parlé dans l'espace francophone. Ressources pour l'enseignement. Paris: Ophrys.

Durand, Jacques/Eychenne, Julien (2004): «Le schwa en français: pourquoi des corpus? ». Corpus [En ligne] 3. http://corpus.revues.org/246 [07.10.2015].

Ferguson, Charles Albert (1975): "Toward a Characterization of English Foreigner Talk". Anthropological Linguistics 17/1: 1-14.

Fougeron, Cécile/Goldman, Jean-Philippe/Frauenfelder, Ulrich Hans (2001): "Liaison and schwa deletion in French: an effect of lexical frequency and competition?". Proceedings of the 7th European Conference on Speech Communication and Technology: 639-642.

François, Denise (1974): Français parlé. Analyse des unités phoniques et significatives d'un corpus recueilli dans la région parisienne. Tome 2. Paris: Selaf.

Gadet, Françoise (1989): Le français ordinaire. Paris: Colin.

Gadet, Françoise (2007): La variation sociale en français. Paris: Ophrys.

Gatti, Aurélia (2008): Repas en famille - épinards. Lyon: Clapi. http://clapi.ish-lyon.cnrs.fr/ [07.10.2015].

Gilmore, Alex (2007): "Authentic materials and authenticity in foreign language learning". Language teaching 40/2. Cambridge, Cambridge University Press: 97-118. doi: 10.1017/S0261444807004144.

Giroud, Anick/Surcouf, Christian (2016) : «De « Pierre, combien de membres avez-vous ?» à « Nous nous appelons Marc et Christian » : réflexions autour de l'authenticité dans les documents oraux des manuels de FLE pour débutants ». Congrès Mondial de Linguistique Française: 1-18. 
Grevisse, Maurice/Goosse, André (2008): Le Bon Usage. Grammaire française. Bruxelles: De Boeck/Duculot.

Hansen, Anita Berit (2000): «Le E caduc interconsonantique en tant que variable sociolinguistique. Une étude en région parisienne ». LINX 42/1: 45-58.

Henzl, Vera (1979): "Foreign talk in the classroom". International Review of Applied Linguistics 17/1: 159-167.

Léon, Pierre (1992/2007): Phonétisme et prononciations du français. Paris: Colin.

Lodge, Robert Anthony (1993): French: from dialect to standard. London/New York: Routledge.

Malécot, André (1975): "French Liaison as a Function of Grammatical, Phonetic and Paralinguistic Variables". Phonetica 32/3: 161-179.

Martinet, André (1974): Le français sans fard. Paris: Presses universitaires de France.

Morin, Yves Charles (1978): "The Status of Mute 'e"”. Studies in French Linguistics 1/2: 79140.

Morin, Yves Charles (1988): "Explaining Schwa in French". In: Slater, Catherine/Durand, Jacques/Bate, Michèle (eds.): Occasional Papers of the University of Essex: French Sound Patterns Changing Perspectives. Colchester, University of Essex: 250-265.

Munro, Murray J. (2013): "Intelligibility". In: Chapelle, Carol (ed.): The Encyclopedia of Applied Linguistics. Chichester, Blackwell: 2732-2739.

Otsuka, Yoko (2006): Repas - conversation entre étudiants. Lyon: Clapi. http://clapi.ishlyon.cnrs.fr/ [07.10.2015].

Rey-Debove, Josette (ed.) (1999): Dictionnaire du français. Paris: Robert/CLE.

Rey-Debove, Josette/Rey, Alain (eds.) (2009): Le Nouveau Petit Robert 2010. Paris: Robert.

Riegel, Martin/Pellat, Jean-Christophe/Rioul, René (1994): Grammaire méthodique du français. Paris: Presses Universitaires de France.

Riegel, Martin/Pellat, Jean-Christophe/Rioul, René (2009): Grammaire méthodique du français. Paris: Presses Universitaires de France.

Rouayrenc, Catherine (2010): Le français oral 1. Les composantes de la chaine parlée. Paris: Belin.

Séguin, Hubert (1989): «Pour une taxinomie de la morphologie verbale en didactique du français ». Études de linguistique appliquée 75: 124-137.

Thévenon, Emmanuel/Tommasini, Isabelle (2008): Repas entre ami(e)s - Kiwi. Lyon: Clapi. http://clapi.ish-lyon.cnrs.fr/ [07.10.2015].

Traverso, Véronique (1985-1986): Conversations familières - visites 1, 2 et 3. Lyon: Clapi. http://clapi.ish-lyon.cnrs.fr/ [07.10.2015].

\section{Corpus}

Abry, Dominique/Fert, Catherine/Parpette, Chantal/Stauber, Julie (2008): Ici 1. Méthode de français. Paris: CLE.

Bérard, Évelyne/Canier, Yves/Lavenne, Christian (1996): Tempo 1. Méthode de français. Paris: Didier/Hatier.

Berger, Dominique/Mérieux, Régine (1994a): Cadences 1. Paris: Hatier/Didier.

Berger, Dominique/Mérieux, Régine (1994b): Cadences 1. Guide pédagogique. Paris: Hatier/Didier. 
Berger, Dominique/Spicacci, Nerina (2000): Accord. Méthode de français niveau 1. Paris: Didier.

Berthet, Annie/Hugot, Catherine/Kizirian, Véronique/Waendendries, Monique (2012): Alter ego + 1. Paris: Hachette.

Courtillon, Janine/Raillard, Sabine (1982a): Archipel 1. Paris: Didier.

Courtillon, Janine/Raillard, Sabine (1982b): Archipel. Livre 1. Livre du professeur. Paris: Didier.

Labascoule, Josiane/Lause, Christian/Royer, Corinne (2004): Rond-point 1. Barcelone/SaintMartin-d'Hères: Difusión/PUG.

Lavenne, Christian/Bérard, Évelyne (2001): Studio 60 niveau 1. Méthode de français. Paris: Didier.

Mérieux, Régine/Loiseau, Yves (2004): Connexions 1. Paris: Didier.

Mimran, Reine/Poisson-Quinton, Sylvie (2012): Zénith 1. Paris: CLE. 